\section{Alzheimer's drugs: Does reducing amyloid work?}

In his Perspective "Treatments for Alzheimer's disease emerge" (6 August, p. 624), D. J. Selkoe asserts that some trials testing potential treatments for Alzheimer's disease have shown "evidence of disease modification." He cites reductions in amyloid plaques (hypothesized to cause cognitive decline) and some modest reductions in cognitive decline shown in four potential drugs that target amyloid. However, hardly any trials have shown an effect, and even the trials with statistically significant results show effects that are too small to be clinically significant or to justify moving forward with the treatments. $\beta$-amyloid antibodies can lower amyloid plaques (extracellular aggregated insoluble $\beta$-amyloid), but available data show that decreasing amyloid plaques does not in itself lead to reduction in cognitive decline.

The data from six phase 2 or 3 trials of the four medications cited by Selkoe are available in peer-reviewed articles. Four trials were stopped for futility and one trial, lecanemab, was negative (1). Only one trial hit its primary endpoint (for donanemab) (2). The negative lecanemab trial did not meet its primary endpoint at 12 months, despite the potential advantage of a protocol change that created an imbalance in $A P O E 4$ carriers, who experience faster cognitive decline. With only $30 \%$ of the treatment cohort composed of APOE4 carriers compared with $71 \%$ of the placebo group, the placebo group would be expected to decline more quickly $(3,4)$. Selkoe claims that one gantenerumab phase 2 trial reduced amyloid and cognitive decline, but both reported phase 3 gantenerumab trials were stopped for futility and had no significant effects on primary or secondary outcomes (5) at 2 years. Selkoe characterizes a small trial of donanemab as "markedly" decreasing amyloid and "significantly" slowing cognitive decline. However, this trial showed only a 3.2-point benefit on a 144-point scale-half the trial team's designated minimally clinically significant effect size (2)—and no significant effects on secondary cognitive and functional outcomes (6). One aducanumab trial, EMERGE (NCT02484547), showed a 0.39 -point (23\%) better outcome for the treatment group on the primary Clinical Dementia Rating (CDR-SB) scale outcome at 18 months, but the identical ENGAGE trial showed a 0.03 -point $(2 \%)$ worsening with treatment (7).

Selkoe speculates several reasons for failures of past trials but ignores what might be the most obvious: The treatment target ( $\beta$-amyloid) itself may be wrong. Just as removing smoke does not extinguish a fire, reducing amyloid plaques may not affect the course of Alzheimer's disease. Certainly, trial data do not support any clinical benefit of amyloid plaque reduction (8). Neither donanemab nor aducanumab trialists reported an association between amyloid reduction and individual participant clinical outcomes $(2,7)$. No comparable published results are available for lecanemab or gantenerumab. Furthermore, the amyloid cascade hypothesis proposes $\beta-$ amyloid aggregation as an early disease trigger, preceding tau phosphorylation and accumulation (9). However, despite reducing amyloid plaques, donanemab failed to lower tau and also increased brain atrophy (10).

Alzheimer's disease antibody trials represent the definitive test of the amyloid hypothesis of Alzheimer's disease. Objective appraisal of the clinical outcomes data suggests more a failure of hypothesis confirmation than successful translation of this disease model.

Madhav Thambisetty ${ }^{1}$, Robert Howard ${ }^{2}, M$. Maria Glymour ${ }^{3}$, Lon S. Schneider

${ }^{1}$ Clinical and Translational Neuroscience Section, Laboratory of Behavioral Neuroscience, National Institute on Aging, Baltimore, MD 21224, USA. ${ }^{2}$ Division of Psychiatry, University College London, London W1T 7NF, UK. ${ }^{3}$ Department of Epidemiology and Biostatistics, University of California, San Francisco, CA 94158, USA. ${ }^{4}$ Department of Psychiatry and the Behavioral Sciences, Department of Neurology, and the USC Alzheimer Disease Research Center, Keck School of Medicine, University of Southern California, Los Angeles, CA 90089, USA *Corresponding author. Email: thambisettym@mail.nih.gov

REFERENCES AND NOTES

1. C. J. Swanson et al., Alzheimers Res. Ther. 13, 80 (2021).

2. M. A. Mintun et al., N. Engl. J. Med. 384, 1691 (2021). 3. K. R. Murphy et al., Neuroimage 78, 474 (2013).

4. J. Qian, R. A. Betensky, B. T. Hyman, A. Serrano-Pozo, Neurology 96, e2414 (2021).

5. S. Ostrowitzki et al., Alzheimers Res. Ther. 9, 95 (2017).

6. A. I. Levey, N. Engl. J. Med. 384, 1762 (2021).

7. U.S. Food and Drug Administration, "Drug Approval Package: Aduhelm (aducanumab-avwa). Statistical Review and Evaluation NDA/BLA \#: 761178" (2021); www.accessdata.fda.gov/drugsatfda_docs/nda/2021/7611780rig1s000StatR_Redacted.pdf.

8. S. F. Ackley et al., BMJ 372, n156 (2021)

9. E. S. Musiek, D. M. Holtzman, Nat. Neurosci. 18, 800 (2015)

10. S. Ayton, Eur. J. Neurol. 28, e67 (2021).

COMPETING INTERESTS

L.S.S. has received consulting or advisory fees within the past 3 years from Cortexyme, Cognition Inc, Eisai, Eli Lilly, IBC Ltd, Merck, Neurim Ltd, Roche/Genentech, Samus, Takeda, UCB; and has received grants or contracts from Biogen, Eisai, Eli Lilly, and Novartis related to the subject matter of this correspondence.

$10.1126 /$ science.abl8366 